\title{
Low-temperature Bessel beam trap for single submicrometer aerosol particle studies
}

\section{Journal Article}

Author(s):

Lu, Jessica W.; Isenor, Merrill; Chasovskikh, Egor; Stapfer, David; Signorell, Ruth (D)

Publication date:

2014-09

Permanent link:

https://doi.org/10.3929/ethz-b-000089579

Rights / license:

In Copyright - Non-Commercial Use Permitted

Originally published in:

Review of Scientific Instruments 85(9), https://doi.org/10.1063/1.4895118 


\section{Low-temperature Bessel beam trap for single submicrometer aerosol particle studies}

Cite as: Rev. Sci. Instrum. 85, 095107 (2014); https://doi.org/10.1063/1.4895118

Submitted: 30 June 2014 . Accepted: 27 August 2014 . Published Online: 16 September 2014

Jessica W. Lu, Merrill Isenor, Egor Chasovskikh, David Stapfer, and Ruth Signorell

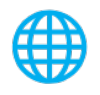

\section{ARTICLES YOU MAY BE INTERESTED IN}

Stability of aerosol droplets in Bessel beam optical traps under constant and pulsed external forces

The Journal of Chemical Physics 142, 154506 (2015); https://doi.org/10.1063/1.4917202

Dynamics of submicron aerosol droplets in a robust optical trap formed by multiple Bessel beams

Journal of Applied Physics 115, 154304 (2014); https://doi.org/10.1063/1.4871540

Dynamic measurements and simulations of airborne picolitre-droplet coalescence in holographic optical tweezers

The Journal of Chemical Physics 145, 054502 (2016); https://doi.org/10.1063/1.4959901

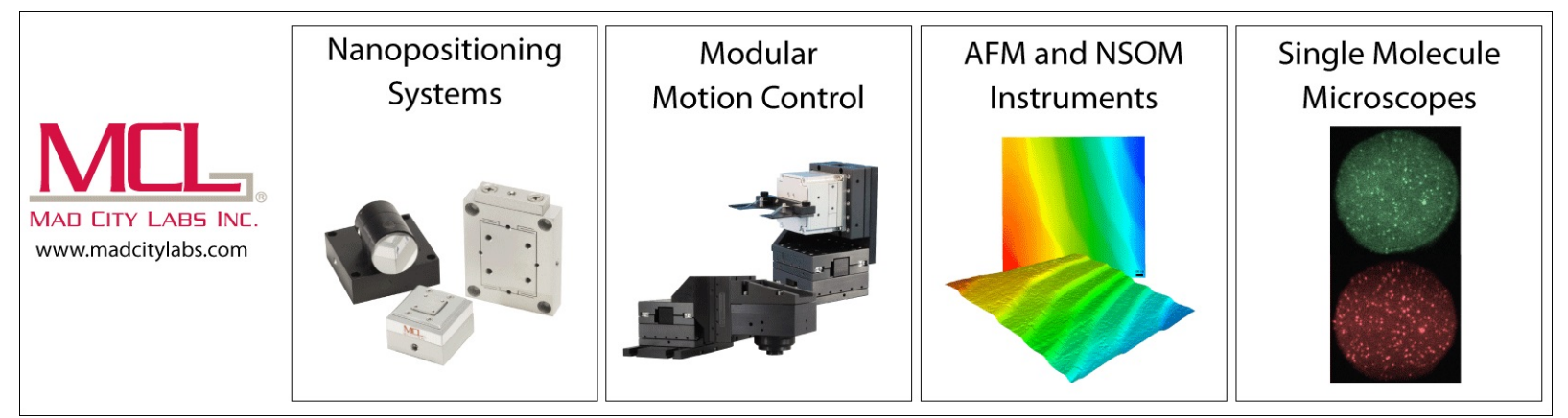




\title{
Low-temperature Bessel beam trap for single submicrometer aerosol particle studies
}

\author{
Jessica W. Lu, ${ }^{1}$ Merrill Isenor, ${ }^{1,2}$ Egor Chasovskikh, ${ }^{1}$ David Stapfer, ${ }^{1}$ and Ruth Signorell ${ }^{1,2}$ \\ ${ }^{1}$ Laboratory of Physical Chemistry, ETH Zürich, Vladimir-Prelog-Weg 2, CH-8093 Zürich, Switzerland \\ ${ }^{2}$ Department of Chemistry, University of British Columbia, 2036 Main Mall, Vancouver, British Columbia V6T \\ 1Z1, Canada
}

(Received 30 June 2014; accepted 27 August 2014; published online 16 September 2014)

\begin{abstract}
We report on a new instrument for single aerosol particle studies at low temperatures that combines an optical trap consisting of two counter-propagating Bessel beams (CPBBs) and temperature control down to $223 \mathrm{~K}\left(-50^{\circ} \mathrm{C}\right)$. The apparatus is capable of capturing and stably trapping individual submicrometer- to micrometer-sized aerosol particles for up to several hours. First results from studies of hexadecane, dodecane, and water aerosols reveal that we can trap and freeze supercooled droplets ranging in size from $\sim 450 \mathrm{~nm}$ to $5500 \mathrm{~nm}$ (radius). We have conducted homogeneous and heterogeneous freezing experiments, freezing-melting cycles, and evaporation studies. To our knowledge, this is the first reported observation of the freezing process for levitated single submicrometer-sized droplets in air using optical trapping techniques. These results show that a temperature-controlled CPBB trap is an attractive new method for studying phase transitions of individual submicrometer aerosol particles. (C) 2014 AIP Publishing LLC. [http://dx.doi.org/10.1063/1.4895118]
\end{abstract}

\section{INTRODUCTION}

Single-particle levitation techniques facilitate the study of aerosol phase transitions and offer critical experimental advantages for the investigation of particle properties which are inaccessible to bulk measurements. Contact-free methods also allow for precise experimental control over particle surroundings, temperature, and relative humidity (RH), without the presence of a surface that could affect the mechanism or kinetics of the phase transition of interest. Suspended single liquid particles may be supercooled to a lower temperature and exist in a metastable state longer than particles that are in contact with a surface since the surface can play a role in nucleation and thus alter the mechanism and rate by which freezing to a crystalline solid occurs. Therefore, single-particle trapping techniques offer the prospect to study phase transitions more precisely by allowing the particle access to non-equilibrium regimes and minimizing perturbations that come from surface contact.

To date, the development of single-particle instrumentation has contributed to an overall deeper understanding of the kinetics and mechanisms of phase transitions in aerosols. Through currently available experimental methods, researchers have systematically explored particle efflorescence and deliquescence, ${ }^{1,2}$ transitions to an ultra-viscous or "glassy phase," ${ }^{,-7}$ and particle freezing, ${ }^{8-15}$ i.e., homogeneous nucleation and crystallization induced by a change, typically a decrease, in the ambient temperature. Fully characterizing aerosol phase transitions and quantifying particle freezing rates are ultimately important for contributing to our understanding of the direct and indirect effect of aerosols in the atmosphere and their overall impact on climate. ${ }^{16-18}$

A suite of different levitation techniques is available to investigate phase transitions of single particles in light of their chemical and physical properties. An instrument that has been extensively used is the electrodynamic bal- ance $(\mathrm{EDB}),{ }^{19}$ in which a charged particle is captured in the electric field produced by two hyperboloidal electrodes and a central ring electrode. One of the first temperaturecontrolled single-particle levitation instruments was a thermostated, cooled EDB apparatus developed by Krämer et al. ${ }^{8}$ that was used to explore the homogeneous ice nucleation rate in single, isolated micrometer-sized water droplets. This version of the cooled EDB offered control over the temperature, $\mathrm{RH}$, composition, and ambient pressure in the environment of a charged aerosol particle. Since then, the technique has been adapted and applied to study the homogeneous freezing of hydrocarbons, ${ }^{12}$ water, ${ }^{8-11,13,15,20,21}$ and mixed watersulfuric acid droplets. ${ }^{14,22}$ The cooled EDB provided the first complete sets of measurements of the freezing kinetics of single particles for these different types of micrometer-sized organic and inorganic particles. However, while the theoretical lower size limit for trapping is on the order of hundreds of nanometers, ${ }^{23}$ in practice the lower size limit in the EDB seems to be $r=2 \mu \mathrm{m}$, and most studies have been performed with much larger particles. ${ }^{4}$ Additionally, a disadvantage of EDBs is that particles must be charged, which could potentially affect the freezing dynamics for smaller particles. ${ }^{15,24}$

Single aerosol particle studies can also be performed in optical traps. Such laser trapping techniques are complementary to the EDB and offer distinct advantages, such as the ability to trap neutral dielectric particles that are smaller in size. The first cooled optical trapping apparatus was developed by Anders et al. ${ }^{25}$ in which the freezing of supercooled water ${ }^{25,26}$ and mixed water-sulfuric acid droplets ${ }^{25}$ immobilized in a focused Gaussian beam ${ }^{27}$ was observed. However, the particles were only able to be trapped on a timescale of minutes. Similarly, Mund and Zellner ${ }^{28}$ constructed an apparatus that coupled optical levitation with Raman spectroscopy to investigate single supercooled, micrometer-sized, mixed water-sulfuric acid droplets on a timescale of hours. ${ }^{29} \mathrm{~A}$ laser 
trapping technique was developed by Taji et al. ${ }^{24}$ that utilized focused counter-propagating Gaussian beams of equal power to trap supercooled water droplets ${ }^{24,30}$ and ice crystals. ${ }^{24}$ Ishizaka et al. ${ }^{31}$ have applied a cooled single-particle trapping apparatus incorporating optical tweezers to the study of crystallization of aqueous ammonium sulfate droplets in air. In addition to these different optical levitation devices, an acoustic trap $^{32,33}$ and a quadrupole trap ${ }^{34}$ have been developed to study the freezing of sulfuric acid/nitric acid and pure sulfuric acid systems, respectively, but typically for larger particles at least $r=20 \mu \mathrm{m}$ in size. While these techniques have contributed valuable insight into the freezing dynamics of supercooled aerosol particles, the practical lower size limit for available cooled optical trapping instruments (for trapping at ambient pressure) also appears to be $r=2 \mu \mathrm{m},{ }^{24}$ and, like with the EDB, most studies have been performed with larger particles.

The development of the counter-propagating Bessel beam (CPBB) trap provides a promising technique that allows researchers to capture and entrain particles down into the submicrometer size range. A Bessel beam possesses a circularly symmetric profile that consists of a central core surrounded by multiple rings. Two orthogonally-polarized CPBBs overlap to form the trapping region, where particles are confined. The CPBB trap possesses unique properties that make it advantageous for the study of single-particle phase transitions: neutral dielectric particles can be trapped, particles can be trapped far from any surface, and the pseudo-nondiffracting beams can reform after an obstacle allowing for trapping of multiple particles. The lower size limit of particles trapped with a CPBB trap appears to be in the hundreds of nanometers, with size limitations coming mainly from the ability to accurately size the particles. Originally developed by Cizmar and coworkers, ${ }^{35-38}$ the technique has been modified and applied by Reid and co-workers ${ }^{39,40}$ and used to study single particles at room temperature. McGloin, Reid, and co-workers have also demonstrated the trapping of submicrometer- to micrometersized particles in air ${ }^{40,41}$ and Cotterell et al. ${ }^{42}$ have used a $405 \mathrm{~nm}$ laser to interrogate the size of a small particle $(r=$ $350 \mathrm{~nm}$ ) trapped using a single Bessel beam against a counter flow of $\mathrm{N}_{2}$. Recently, we have performed studies exploring the ultraviscous behavior of single sucrose droplets near the glass transition RH region for submicrometer-sized particles $(r=700 \mathrm{~nm})$ at room temperature. ${ }^{43}$ The CPBB trap can thus be used to extend the size limit for studying phase transitions in particles below $r=2 \mu \mathrm{m}$ and into the submicrometer size range.

We seek to improve the capability of the CPBB trap to study the phase transitions of submicrometer- to micrometersized particles by combining existing CPBB trap technology with temperature control into a single apparatus. Particles are trapped far from surfaces in the CPBB trap and this can minimize temperature gradients and convection that could lead to particle instability. The size (and therefore, volume and surface area) of single aerosol particles is determined from elastic light scattering measurements. We show first examples of homogeneous and heterogeneous freezing, freezingmelting cycles, and evaporation of single particles. In the following work, we explore single, submicrometer-sized water and hydrocarbon aerosols that are stably trapped on the order of hours in a CPBB trap across a wide temperature range $(226-330 \mathrm{~K})$ relevant to planetary and lunar atmospheres (e.g., Earth, ${ }^{44-47}$ Mars, ${ }^{48}$ Titan $^{49-51}$ ). Studying finemode $(r<1 \mu \mathrm{m})$ water and hydrocarbon aerosols at cold temperatures may help us to better understand, respectively, cirrus clouds that form in the upper troposphere/lower stratosphere, as well as organic aerosols in the lower troposphere with anthropogenic origins. ${ }^{46,47}$

\section{INSTRUMENTAL SETUP}

\section{A. Single-particle levitation chamber}

Our design of the CPBB trap levitates and confines submicrometer- to micrometer-sized aerosol particles in a temperature-controlled environment. The apparatus consists of a vacuum chamber for thermal insulation and an inner trapping chamber cooled by a thermostated refrigerated circulation unit. A schematic of the single-particle levitation apparatus is shown in Figure 1. The stainless steel vacuum chamber (custom designed, Hositrad Vacuum Technology), with a diameter of $24 \mathrm{~cm}$ and volume of $7.2 \times 10^{3} \mathrm{~cm}^{3}$, is attached to a raised aluminum breadboard that is secured on an optical table (Thorlabs, Inc.). On the vacuum chamber, flanges labeled A, $\mathrm{B}$, and $\mathrm{C}$ contain $50 \mathrm{~mm}$ diameter BK7 windows (UQG Optics) for passage of the counter-propagating Bessel beam arms and collection of elastically scattered light from the aerosol particle. The vacuum chamber is pumped by a small turbomolecular pump (Pfeiffer Vacuum) that maintains pressures between $1 \times 10^{-5}$ and $1 \times 10^{-6}$ mbar over a wide temperature range (226-330 K). The pump is connected via a flexible tube through a side port of the vacuum chamber and rests on the ground to minimize vibrations in the trapping chamber.

The copper trapping cell located at the center of the vacuum chamber is constructed of oxygen-free high thermal

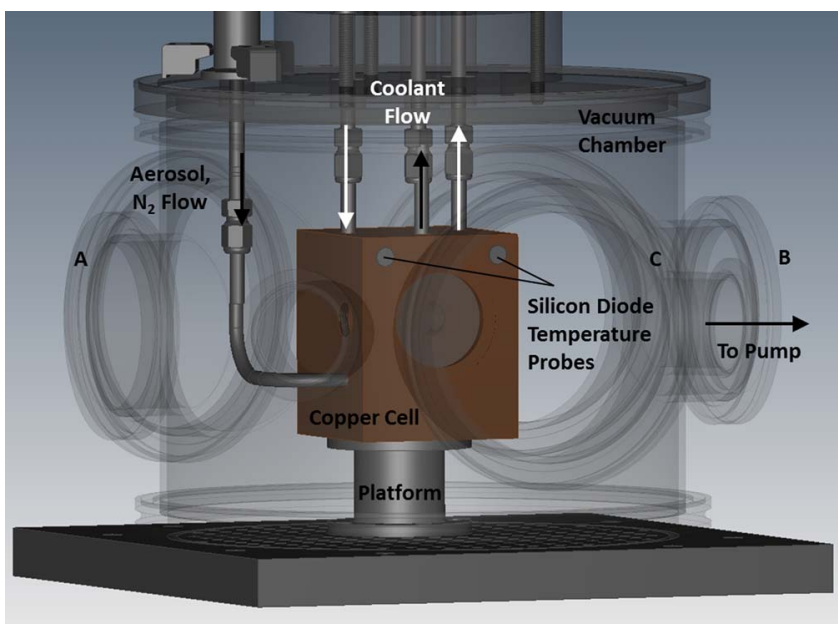

FIG. 1. A schematic representation of the single-particle trapping apparatus with vacuum chamber and copper trapping cell with temperature control. The color of the vacuum chamber walls has been lightened to show the positioning of the copper cell in the chamber. The copper cell is placed on an insulating platform attached to the breadboard. The flow of aerosols and $\mathrm{N}_{2}$ (dry or humidified) is introduced via a stainless steel tube into the trapping region. The coolant is cycled through the copper cell through tubing connected to the trap that leads to a thermostated refrigerated circulator. 
conductivity copper and machined at an in-house machine shop (ETH). As shown in Fig. 1, the copper cell is cooled by a closed-cycle refrigerated circulator (Lauda, Proline RP 1290) which introduces an oil-based coolant (Atelier für Metallbau, Wärmeträger Fluid WTF 190) through the cell via stainless steel tubes attached to the main body of the trapping cell. The copper cell is positioned on an insulating platform attached to the breadboard. Two silicon diode temperature sensors (Lakeshore, DT-670 Series) are placed at the top corners of the copper cell. Each sensor has an absolute uncertainty of $\pm 0.025 \mathrm{~K}$, and during experiments, the difference in the measured temperature between the two probes on the copper cell is $0.03 \mathrm{~K}$. The difference between the temperature in the trapping region and the probe measurement of the copper cell is approximately $\pm 0.5 \mathrm{~K}$, as measured by a Type $\mathrm{K}$ thermocouple (Omega) in the trapping region (this will be replaced by a silicon diode temperature sensor). However, the fluctuation of the temperature reported in the trapping region appears to be appropriately represented by this probe. For example, at $228.0 \mathrm{~K}$, the fluctuation in the measured temperature over a period of $20 \mathrm{~min}$ is $\pm 0.03 \mathrm{~K}$. The fluctuation in the measured temperature of the copper trap is to be expected due to the periodic internal temperature fluctuations of the thermostated refrigerated circulation unit at these set temperatures. The experimental temperature range for the measurements described in this work spans from $228.0 \mathrm{~K}$ to $294.0 \mathrm{~K}$. The maximum cooling rate for the copper cell as measured by the silicon diode temperature probes is $1 \mathrm{~K} / \mathrm{min}$.

A cross-sectional view of the copper trapping chamber and the relative position of the CPBBs is given in Figure 2. The cell consists of a central trapping region that has a volume of $2.3 \mathrm{~cm}^{3}$ and, combined with the volume for each Bessel beam arm, has a total volume of $5.1 \mathrm{~cm}^{3}$. The CPBBs pass through $15 \mathrm{~mm}$ diameter BK7 windows (Edmund Optics) with $532 \mathrm{~nm}$ coating to minimize scattered light. The flow of aerosols and ultrahigh purity $\mathrm{N}_{2}$ (dry or humidified) is introduced into the cell perpendicular to the plane of the

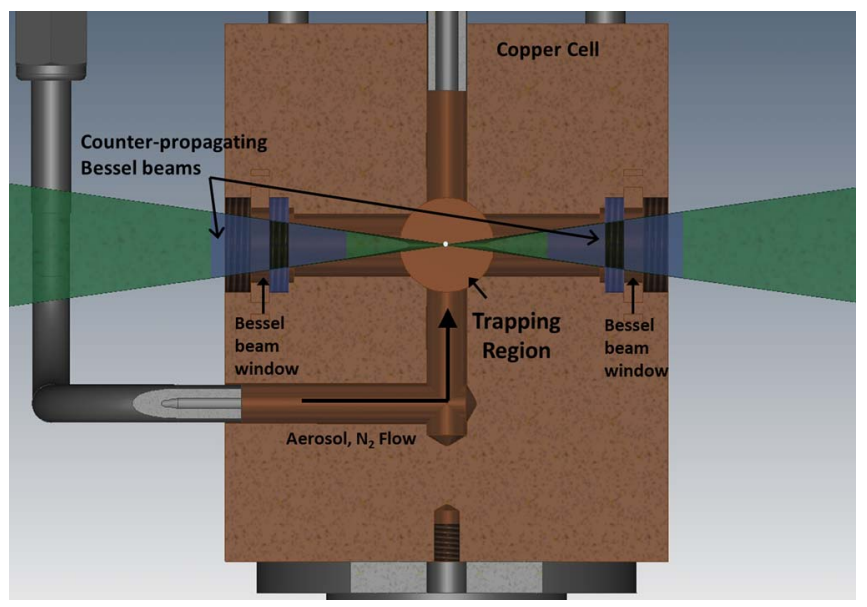

FIG. 2. Cross-sectional view of the copper trapping cell in the plane of the counter-propagating Bessel beams. The orthogonally-polarized beams enter the trapping chamber through the $532 \mathrm{~nm}$-coated BK7 Bessel beam windows. The flow for aerosols and $\mathrm{N}_{2}$ (dry and humidified) into the cell is oriented perpendicular to the plane of the counter-propagating Bessel beams. The aerosol particles are trapped in the center of the trapping region. trapping beams through stainless steel tubing that lead directly to the trapping region. A pair of mass flow controllers (Bronkhorst, EL-FLOW Mass Flow Meters, Series F-201CV) gives a maximum flow rate of $100 \mathrm{~cm}^{3} / \mathrm{min}$, but is typically set to $0.25-10 \mathrm{~cm}^{3} / \mathrm{min}$ during experiments. For certain experiments, the $\mathrm{N}_{2}$ was initially passed through a copper coil immersed in a separate refrigerated circulator (Fisher Scientific, Isotemp 3016) cooled to $273 \mathrm{~K}$. Thus, a constant $\mathrm{N}_{2}$ flow is maintained through the interior of the copper cell and the trapping region, keeping the pressure at approximately $1 \mathrm{~atm}$.

\section{B. The counter-propagating Bessel beam trap}

In the present work, we use a CPBB trap that is created through the overlap of two orthogonally polarized counterpropagating arms. The optical setup depicted in Figure 3 is similar to the setup described in Carruthers et al..$^{39}$ and $\mathrm{Lu}$ et al. ${ }^{43}$ The beam waist of the incident Gaussian beam from the continuous wave laser (Laser Quantum, Opus 3, $532 \mathrm{~nm}$, typical laser power $1900 \mathrm{~mW}$ ) is expanded by telescope from $2 \mathrm{~mm}$ to approximately $4 \mathrm{~mm}$. The expanded beam then passes through an axicon with an opening angle of $176^{\circ}$ (Altechna). After the axicon, the beam is split into two orthogonally polarized components using a polarization beam splitter cube. The CPBBs overlap within the copper trapping cell to form the "trapping region" within which single particles are captured. The radius of the Bessel beam core was $2 \mu \mathrm{m}$ and the power of the core in the trapping region was $8 \mathrm{~mW}$ for these experiments. Stable trapping is achieved through contributions from both the radial forces (strong transverse "gradient forces" determined by the field gradients) and the axial forces (longitudinal "scattering forces" resulting from radiation pressure). ${ }^{52}$ The $\mathrm{CPBB}$ trap has the ability to trap

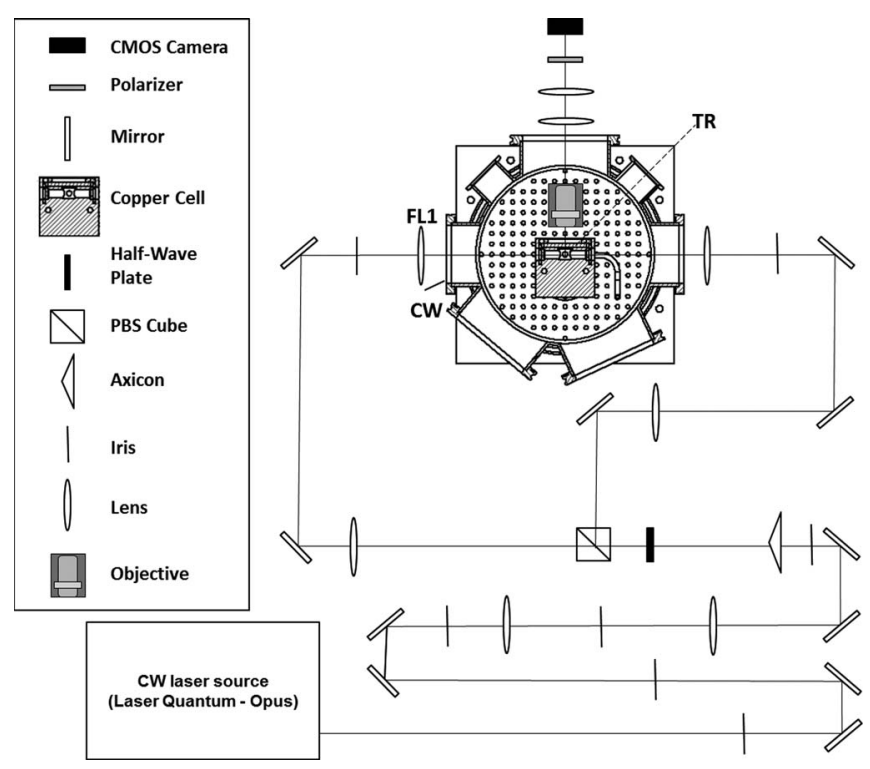

FIG. 3. Schematic view of the counter-propagating Bessel beam (CPBB) trap with imaging and data collection system. The CPBBs overlap in the trapping region (TR). The distance between focusing lens 1 (FL1) and the vacuum chamber window $(\mathrm{CW})$ is $2.5 \mathrm{~cm}$. The distance between the $\mathrm{CW}$ and the TR in the center of the copper cell is $14.5 \mathrm{~cm}$. 
submicrometer particles far from any surface and on the order of hours to days.

\section{Particle generation}

Individual aerosol particles are captured in the CPBB trap from a plume of dispersed aerosols generated by a medical nebulizer (Pari, PARI Boy SX). While the median particle radius generated is $1.75 \mu \mathrm{m}$, the distribution of particle radii spans a broad range, from hundreds of nanometers to several micrometers. In the current work, we generated aerosols from hexadecane (ABCR Chemicals, 99\%), dodecane (Acros Organics, 99\%), and milipure water at room temperature. The chemicals were used without further purification.

\section{Particle imaging/data collection system}

As shown in Fig. 3, the elastically scattered light from the particle exits the cell through a $50 \mathrm{~mm}$ diameter BK7 window and is collected and focused using a $20 \times$ objective (Mitutoyo, Plan Apo Infinity-Corrected Long WD Objective, N.A. $=0.42$ ) oriented at $90^{\circ}$ relative to the forward scattering direction and located in the scattering plane. The objective is attached to a XYZ Gothic-arch bearing translational stage (Newport, \#9061-XYZ-PPN-M) equipped with Picomotor actuators (Newport, \#8302) to provide in vacuo translational motion. The scattered light then passes through a series of lenses and a linear polarizer, to limit light collection to polarized light oriented perpendicularly to the scattering plane, and is recorded with a standard CMOS camera (Thorlabs, DCC1645C, $1280 \times 1024$ pixels) either in snapshots or as a video, typically at 10 frames/s. The electronic components are controlled by a personal computer (PC) with data acquisition capability, and the user interface is programmed in LabVIEW.

\section{EXPERIMENTAL RESULTS}

We have used the low-temperature CPBB trap with temperature control to trap single, supercooled hexadecane, dodecane, and water aerosols. In this section, we discuss preliminary freezing results for each of these substances. Particles remain trapped for timescales of minutes to hours. Particles ranging in size from $\sim 450 \mathrm{~nm}$ to $5500 \mathrm{~nm}$ (radius) have been successfully trapped and sized in our current setup. While it is likely that smaller particles have been trapped, we are limited in our current ability to size particles below $\sim 400 \mathrm{~nm}$.

Particle sizing was performed using the images of elastically scattered, perpendicularly polarized light collected over an angular range of $\sim 66^{\circ}-114^{\circ}$. The particle radius $(r)$ was determined by fitting experimentally obtained phase functions (from the measured scattering images), to simulated phase functions using Mie theory. ${ }^{53}$ The simulation needs the refractive index (RI), scattering angles, laser polarization, and a trial size range as input parameters to calculate phase functions and corresponding particle radii. The "best-fit" phase function and particle $r$ were determined by visual inspection of the quality of the fit and by finding the calculated minimum "error" (the mean of the absolute difference between the experimental and calculated phase functions). The error on all sizes is approximately $100-150 \mathrm{~nm}$, which is typically the size difference between two fits of nearly identical quality and error. ${ }^{43}$ This error also encompasses the quality of the snapshot and corresponding phase function and the uncertainty in the RI and scattering angles.

Accurate RI values are necessary for appropriate size determination of the trapped particles. RI values vary as a function of temperature and wavelength. Since RI literature data at our experimental temperature and wavelength are unavailable, "corrections" for temperature and wavelength were made to available literature RI values to obtain the appropriate RI for size analysis for each substance. ${ }^{54,55}$ Initially, the temperature correction for hexadecane and dodecane was estimated by fitting a second-order polynomial to available RI data at a single wavelength $(632.8 \mathrm{~nm})$, then deriving an expression that was used to extrapolate the RI to lower temperatures. Assuming a similar relationship between RI and temperature at $589.3 \mathrm{~nm}$ and $632.8 \mathrm{~nm}$ for the two hydrocarbons, a second polynomial expression was derived from a single RI datapoint at $589.3 \mathrm{~nm}$. Subsequently, the hexadecane and dodecane wavelength dispersion in the RI at $532 \mathrm{~nm}$ was determined using the Cauchy relationship, utilizing the extrapolated RI data at $632.8 \mathrm{~nm}$ and $589.3 \mathrm{~nm}$ at the desired temperature. ${ }^{56}$ The resulting estimated RI's of hexadecane and dodecane are $1.445(532 \mathrm{~nm}, 284.2 \mathrm{~K})$ and 1.451 (532 $\mathrm{nm}, 252.0 \mathrm{~K})$, respectively. Similarly, the RI value for supercooled water at $228.0 \mathrm{~K}$ was first approximated at both $632.8 \mathrm{~nm}$ and $589.3 \mathrm{~nm}$ through a fifth-order polynomial $\mathrm{fi}^{57}$ of the available literature data for each wavelength. ${ }^{55,56,58}$ Again applying the Cauchy equation, the estimated RI value for supercooled water is $1.327(532 \mathrm{~nm}, 228.0 \mathrm{~K})$. For each of these substances, the imaginary part was assumed to make a negligible contribution at $532 \mathrm{~nm}$.

\section{A. Hexadecane}

Hexadecane was used as a major test substance in our system due to its ease of trapping, relatively high melting point $(291.27 \mathrm{~K})^{55}$ and low vapor pressure (approximately $0.000294 \mathrm{~mm} \mathrm{Hg}){ }^{59}$ All hexadecane particle studies were performed across the temperature range $286.2-287.5 \mathrm{~K}$. These temperatures represent supercooling of hexadecane particles by approximately $3.8-5.0 \mathrm{~K}$. For all hexadecane measurements, we used a continuous dry $\mathrm{N}_{2}$ flow at a rate of $0.25-4.0 \mathrm{~cm}^{3} / \mathrm{min}$. The $\mathrm{N}_{2}$ was passed through a copper coil immersed in a refrigerated circulator set at $286.15 \mathrm{~K}$ before entering the cell. The higher flow rate was required initially to remove the excess particles from the cell. Once only the trapped particle remained, the flow was reduced as low as $0.25 \mathrm{~cm}^{3} / \mathrm{min}$ during the remainder of the experiment.

The freezing times for 18 supercooled hexadecane particles are plotted in Figure 4 with respect to temperature. The freezing times represent the length of time that particles were trapped at the stated temperature before freezing. The sizes of the particles displayed in the plot range from approximately $r=800 \mathrm{~nm}$ to $r=5500 \mathrm{~nm}$. Fig. 4 shows that on average the particles freeze faster at the lower cell temperatures. This 




FIG. 4. The observed homogeneous freezing times for supercooled hexadecane particles ( $r=800$ to $r=5500 \mathrm{~nm}$ ) as a function of the cell temperature.

trend is to be expected because there is a strong temperature dependence on the rates of homogeneous nucleation, according to the classical theory of nucleation. ${ }^{60}$ Note that the spread in freezing times for similar temperature values comes from the variation in particle size. According to nucleation theory: ${ }^{61}$

$$
-\frac{d \ln P(t, T, r)}{d t}=J_{V}(T) V(r)+J_{s}(T) S(r) .
$$

$P(t, T, r)$ is the probability that droplets of radius $r$ are still liquid at time $t[P(0)=1] . V(r)$ and $S(r)$ are the particle volume and the surface area, respectively, and $J_{V}(T)$ and $J_{S}(T)$ are the corresponding freezing rate constants. Previous studies have shown that a decrease in temperature by $1 \mathrm{~K}$ can increase the freezing rate for water by nearly two orders of magnitude. ${ }^{15}$

Typical scattering images taken before and after freezing are shown in Figure 5. For the large particle displayed in Figs. 5(a) (liquid) and 5(b) (solid), there is a clear difference in the scattering pattern before and after freezing. The light scattered from the liquid particle has a regular striped


FIG. 5. A large $(r=5042 \mathrm{~nm})$ supercooled hexadecane particle at $287.5 \mathrm{~K}$ before (a) and after (b) freezing. A small ( $r=924 \mathrm{~nm}$ ) supercooled hexadecane particle at $286.9 \mathrm{~K}$ before (c) and after (d) freezing. pattern while for the solid particle, the scattering pattern is irregular, changes with time, and in general the integrated intensity of the scattered light is more intense than the intensity observed for the liquid particle. ${ }^{12,25}$ Once nucleation occurs, the droplet loses its spherical symmetry, the characteristic stripes disappear, and the integrated intensity of the scattered light increases. ${ }^{12,25}$ These effects were previously observed by Leisner and co-workers in freezing studies of water ${ }^{10,11,13,15}$ and $n$-alkanes (pentadecane and heptadecane), ${ }^{12}$ by Anders et al. ${ }^{25}$ in the freezing of water and water-sulfuric acid droplets, and by Mund and Zellner ${ }^{62}$ in their studies on the homogeneous nucleation of single watersulfuric acid micro-droplets. This sudden change in the properties of the scattering image is what is used to pinpoint the freezing time for particles. For smaller particles, such as the particle shown in Figs. 5(c) (liquid) and 5(d) (solid), the change in the scattering pattern is often less pronounced. However, there is still a clear increase in the scattering intensity at the time of freezing.

An attractive feature of the CPBB trap is the possibility of multiple particle trapping. The CPBB trap is able to trap multiple particles along the axial direction in the trapping region due to the self-healing properties of the Bessel beam. When a particle is trapped, constructive interference of the laser beam after the obstacle occurs to reform the Bessel beam, thus enabling additional particles to be trapped. Figure 6 shows as an example, a frozen hexadecane particle and a supercooled liquid hexadecane particle trapped next to each other tens of micrometers apart. The image in Fig. 6 illustrates again the differences observed in the scattering patterns for solid vs. liquid particles. With the capability of the CPBB trap to trap multiple particles, it may be possible to observe the freezing of several particles trapped simultaneously. We have shown that simultaneous trapping of $\sim 10$ droplets is feasible.

A substantial problem is that the particles often fall out of the trap immediately after freezing. Although the crystal structure and crystal habit of the frozen hexadecane particle have not been directly probed, bulk hexadecane is known

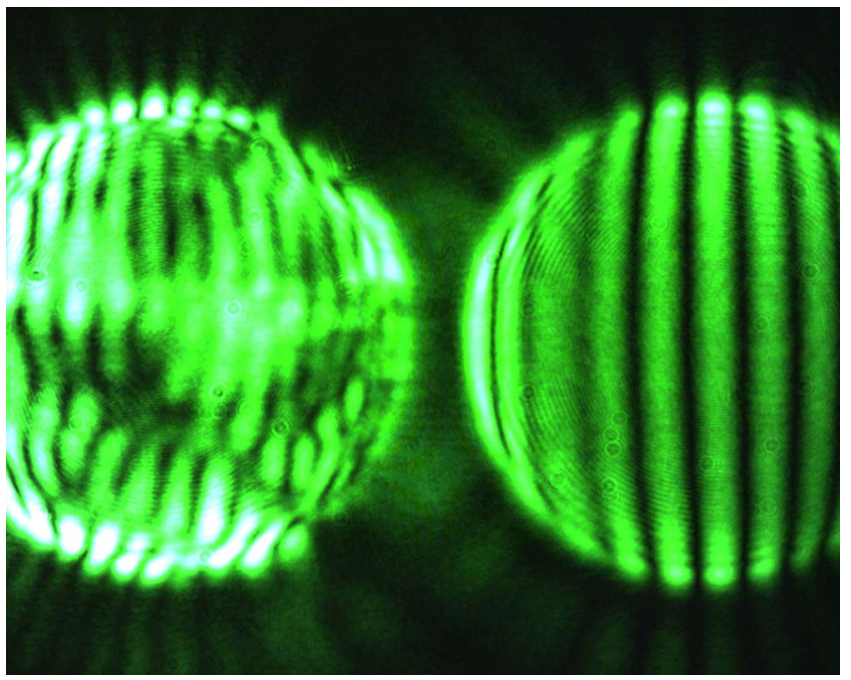

FIG. 6. The CPBB trap can trap multiple particles, as shown by these two hexadecane particles at $287.5 \mathrm{~K}$. The particle on the left is frozen, while the particle on the right is a supercooled liquid. 
to nucleate into the triclinic phase via a transient rotator phase $^{12,63}$ and has a crystal habit that resembles flat, elongated needles. ${ }^{64}$ The crystallized hexadecane particles may fall out of the trap due to the fact that the RI and the shape of the particle suddenly changes during the phase transition, thus altering the balance of forces experienced by the droplet/crystalline solid in the trapping region. ${ }^{31}$ Trapped crystalline particles tend to rotate rapidly, which may be due to a torque given by the radiation pressure of the counter-propagating beams. ${ }^{24}$

We have also performed freezing-melting cycles on single hexadecane particles in the CPBB trap. Starting with either a liquid or frozen particle, we have cycled hexadecane particles between the solid and liquid phase by rapidly decreasing and increasing, respectively, the temperature of the cell below and above the melting point across an overall temperature range of $\sim 10 \mathrm{~K}$. Five particles have been cycled in such a way, with four particles cycled in a solid-liquid-solid cycle and one particle that was frozen 6 times and melted 5 times (total trapping times of $75 \mathrm{~min}$ ). A sample video [see Figure 7 (Multimedia view)] shows one freezing and one melting event for the latter particle. At the start of the video, the particle is a liquid. Decreasing the temperature in the cell causes the particle to freeze at a time of 1:24 in the video. It then melts at a time of 3:33 after increasing the cell temperature.

Several instances of heterogeneous freezing initiated through contact with another particle have been observed for hexadecane particles. Figure 8 shows an example of contact freezing when a larger, supercooled hexadecane particle ( $r=4550 \mathrm{~nm}, T=284.2 \mathrm{~K}$ ) collides with a smaller, supercooled hexadecane particle of unknown size (shadow at the right of the large particle, labeled with an arrow). The small particle provides the contact surface that lowers the free energy barrier to nucleation for the larger droplet. ${ }^{65}$ Once the collision has occurred, freezing of the supercooled hexadecane droplet can be initiated (Fig. 8(b)) and completed

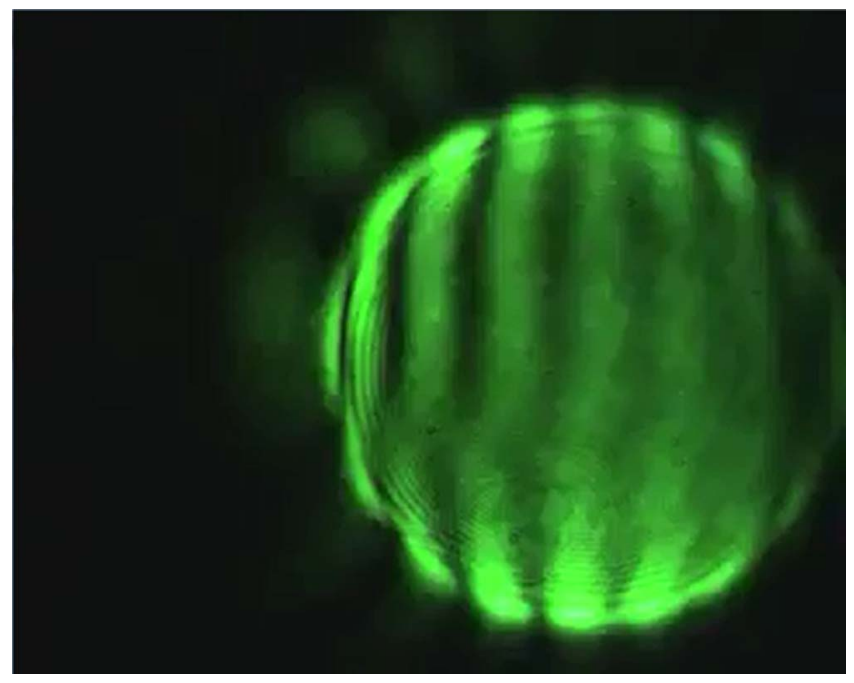

FIG. 7. The video shows an example of a freezing-melting cycle with a supercooled liquid hexadecane particle $(r=2115)$. The particle freezes at 1:24 and melts at 3:33 after the temperature is decreased below and increased above the $T_{\mathrm{m}}$, respectively. The cell temperature is $286.2 \mathrm{~K}$. (Multimedia view) [URL: http://dx.doi.org/10.1063/1.4895118.1]
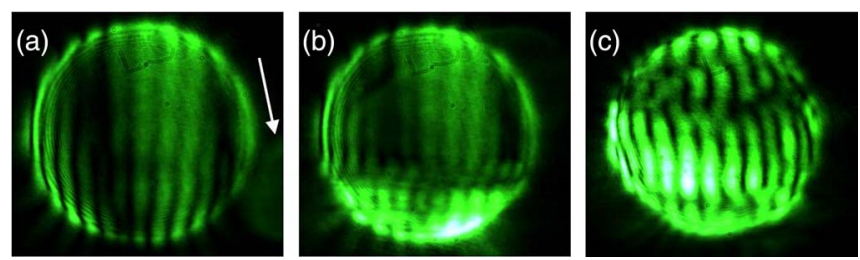

FIG. 8. A supercooled hexadecane particle $(r=4550 \mathrm{~nm})$ before (a), during (b), and after (c) freezing due to contact (through collision) with another supercooled hexadecane particle at $284.2 \mathrm{~K}$. The white arrow in image (a) points to the incoming small particle. The time duration between frames is $0.16 \mathrm{~s}$.

(Fig. 8(c)) nearly instantaneously $(\approx 0.32 \mathrm{~s})$. This behavior is representative of all the freezing events observed for particles that froze heterogeneously through contact freezing.

The evaporation of hexadecane particles in a CPBB trap has also been studied for supercooled hexadecane droplets (data not shown). For that purpose, we monitored the size change of the droplets during evaporation by recording a video of the elastically scattered light from the particle. The hexadecane particles evaporate while trapped under a low dry $\mathrm{N}_{2}$ flow $\left(0.25-4.0 \mathrm{~cm}^{3} / \mathrm{min}\right.$.), which results in a continuous size decrease. For example, particles trapped at a size of $r=3500 \mathrm{~nm}$ typically shrink to around $r=950 \mathrm{~nm}$ within 30 min. Although particles that were smaller than $r \approx 1500 \mathrm{~nm}$ often became less stable as they evaporated, it was possible to monitor the size decrease of droplets down into the submicrometer size range. This opens up the possibility to study evaporation kinetics of droplets at different temperatures.

\section{B. Dodecane}

We have also observed freezing for a small number of supercooled dodecane particles. The melting point for bulk dodecane is $263.58 \mathrm{~K} .{ }^{55}$ All supercooled dodecane liquid particles were trapped at $258.1 \pm 0.2 \mathrm{~K}(5.5 \mathrm{~K}$ supercooled $)$ and further cooled to as low as $252.5 \pm 0.2 \mathrm{~K}(11.0 \mathrm{~K}$ supercooled) before freezing. A dry $\mathrm{N}_{2}$ flow was used during experiments at a flow rate of $0.25-8.0 \mathrm{~cm}^{3} / \mathrm{min}$. Dodecane particles were more difficult to remove from the cell compared to the hexadecane particles, and thus a higher flow rate was required. The $\mathrm{N}_{2}$ flowed through a copper coil immersed in a chiller at a temperature near $273 \mathrm{~K}$ before reaching the cell. We observed homogeneous freezing in less than $60 \mathrm{~s}$ for particles ranging in size from $r=1700 \mathrm{~nm}$ to $r=4700 \mathrm{~nm}$. Like hexadecane, the crystal structure of bulk frozen dodecane is triclinic, and it is assumed that the crystal structure of the frozen dodecane particle is also triclinic. ${ }^{66}$ The crystal habit of dodecane is also expected to be long, flat, and needle-like, as for hexadecane. ${ }^{64}$

Figure 9 shows images of a dodecane particle before (Fig. 9(a)) and after (Fig. 9(b)) freezing. A short video of this freezing is available for viewing [see Figure 10 (Multimedia view)]. The video was started at approximately the same time that the temperature of the cell had stabilized to $252.6 \mathrm{~K}$, thus the time of freezing in the video is approximately the freezing time for the particle at this temperature (time of 0:56 in the video). As for hexadecane, the solid phase is characterized by an irregular scattering pattern and an increase in 

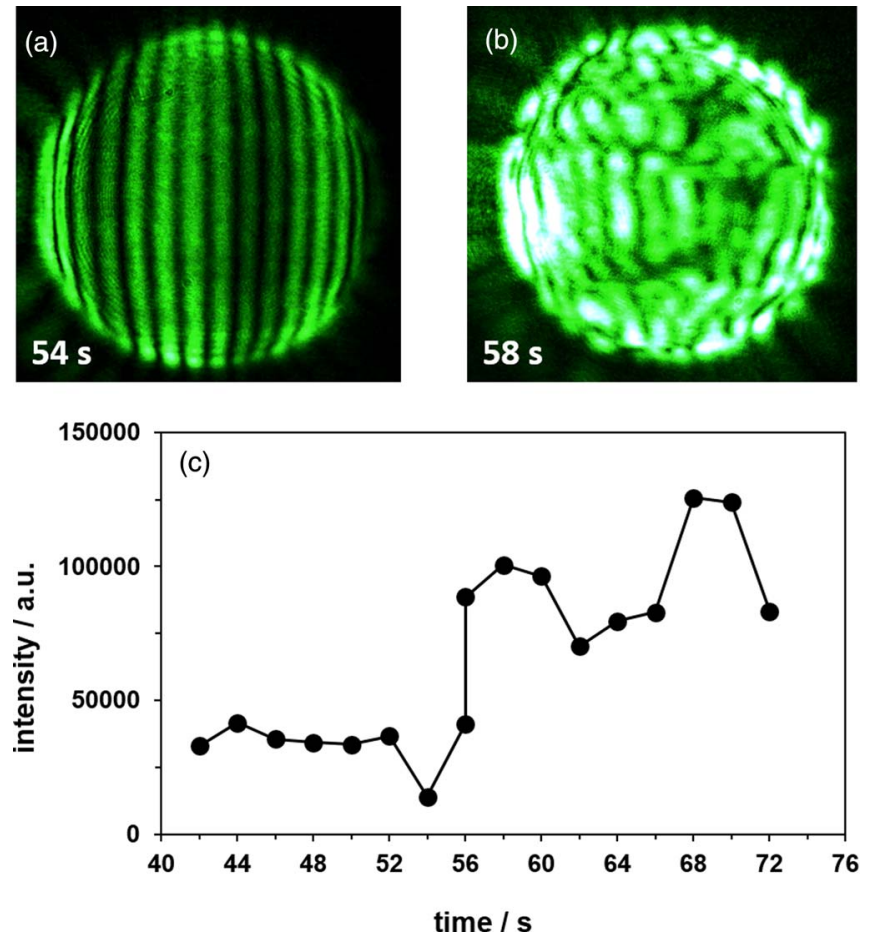

FIG. 9. A dodecane particle ( $r=4180 \mathrm{~nm}$ ) before (a) and after (b) freezing at $252.6 \mathrm{~K}$. (c) The scattering intensity of the perpendicularly polarized light with respect to time. Freezing occurred at $\mathrm{t}=56 \mathrm{~s}$.

scattering intensity. Fig. 9(c) shows the intensity of the perpendicularly polarized scattered light with respect to time. There is a marked increase in the integrated scattering intensity at the time of freezing, such that the scattered light from the solid particle is approximately twice as intense as for the supercooled dodecane droplet. These observations are similar to our observations for hexadecane freezing and previous reports in the literature. ${ }^{12,26}$ In addition, consecutive freezing and melting cycles are also possible for dodecane. The cycles

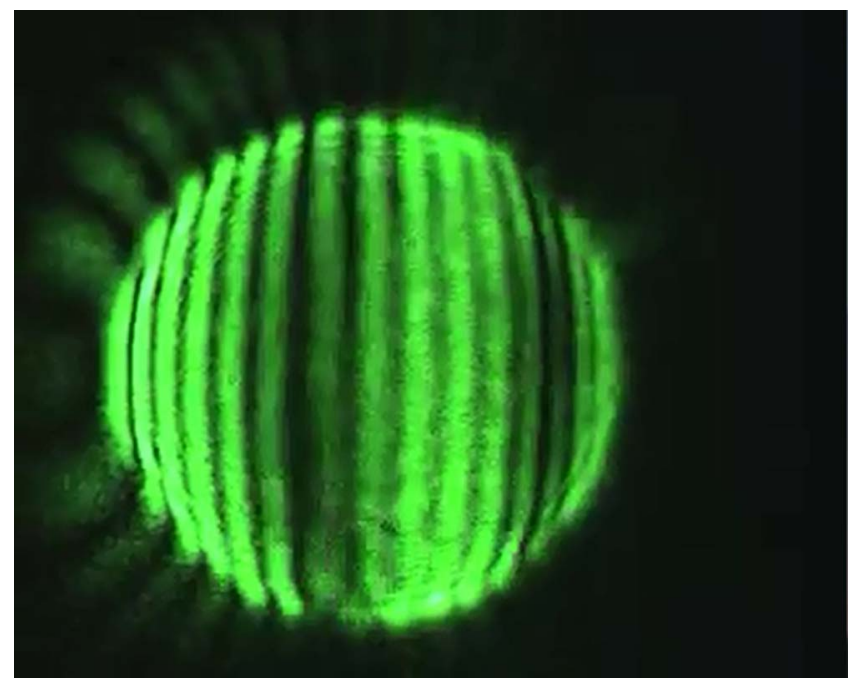

FIG. 10. An example of a freezing-melting cycle with a supercooled liquid dodecane particle $(r=4180 \mathrm{~nm})$. The particle freezes at 0:56 in the video. The cell temperature is $252.6 \mathrm{~K}$. (Multimedia view) [URL: http://dx.doi.org/10.1063/1.4895118.2] were performed in the same manner as for hexadecane, by increasing and decreasing the temperature of the cell above and below the melting point of dodecane to melt and freeze, respectively, the supercooled droplets.

Interest in studying the freezing of hydrocarbon aerosols is motivated in part by the relevance of hydrocarbons to planetary/lunar atmospheres, as well as their importance as "model" nonpolar organic molecules. Previous investigations into aliphatic hydrocarbon $\left(\mathrm{C}_{\mathrm{n}} \mathrm{H}_{2 \mathrm{n}+2}\right)$ aerosol freezing have been performed using both single-particle and ensemble experimental techniques on particles across a broad size range $(r=4 \mathrm{~nm}-60 \mu \mathrm{m})$. An EDB study by Weidinger et al. ${ }^{12}$ on single aerosol particles $(r=60 \mu \mathrm{m})$ examining the freezing of $n=14-17$ hydrocarbons has hinted at the possibility of a surface freezing mechanism for $n=$ odd hydrocarbons. This result was corroborated by Modak et al.'s ${ }^{67}$ combined experimental and computational study on an ensemble of rapidly cooling $n=8,9$ hydrocarbon nanodroplets $(r=4-9 \mathrm{~nm})$ that also indicated a surface freezing mechanism, albeit with a different rate-limiting step. Results from previous work in our research group investigating the freezing of nanometer-sized $(r=50-100 \mathrm{~nm})$ short-chain hydrocarbon aerosols $(n=1-5)$ have been less conclusive. From these ensemble studies with a bath gas cooling cell, it is not clear whether a surface- or volume-freezing mechanism dominates. ${ }^{48-51,61,68-70}$ Furthermore, it is important to notice that, in all these studies, there is a broad size range of aerosols missing that spans nearly three orders of magnitude: $r=200 \mathrm{~nm}-10 \mu \mathrm{m}$. Important questions concerning the freezing of hydrocarbon aerosols remain outstanding, i.e., for particles of which sizes is surface freezing important? How does this change with $n$, if at all? With the CPBB trap, it is in principle possible to begin to address these questions and bridge the knowledge gap of the freezing mechanisms and kinetics for hydrocarbon aerosols.

\section{Water}

The freezing of supercooled water particles at a cell temperature of $228.05 \pm 0.03 \mathrm{~K}$ was also observed. All particles were trapped at this temperature and the temperature setting remained constant throughout all experiments. This temperature represents water particles that were supercooled by $45.1 \mathrm{~K}$. We typically used a humidified flow $(95 \% \mathrm{RH}$ at $294.15 \mathrm{~K}$ ) at a rate of $3.0-5.0 \mathrm{~cm}^{3} / \mathrm{min}$ to try to minimize particle evaporation. At these temperatures, the crystal habit of frozen water is expected to resemble a hexagonal prism that possess a hexagonal crystal structure. ${ }^{71}$

There have been several previous studies of freezing for suspended supercooled water particles. Leisner and coworkers ${ }^{10,11,13,15}$ have studied the homogeneous nucleation of water droplets in a cooled EDB for particles that were on the order of tens of micrometers in radius. They observed homogeneous freezing at temperatures ranging from approximately $236 \mathrm{~K}$ to $238 \mathrm{~K}$. Taji et $_{\text {al. }}{ }^{24}$ have also trapped single liquid and solid water particles in an optical trap. Homogeneous freezing was observed below $233 \mathrm{~K}$ for supercooled water droplets that were typically between $r=10$ and $r=20$ $\mu \mathrm{m}$ in size. The escape times of liquid particles in their trap were on the order of minutes. 
Our current setup allows for stable trapping of much smaller particles compared to previous studies; i.e., down into the submicrometer size regime. Though the evaporation of water droplets limits the time particles remain in the trap, we have been able to trap supercooled water particles for up to $16 \mathrm{~min}$ at a temperature of approximately $228 \mathrm{~K}$. This temperature is below the homogeneous nucleation temperature $(\approx 235 \mathrm{~K} \text { for bulk water })^{72}$ and above the crystallization temperature $(\approx 155 \mathrm{~K})$, the boundary temperature at which amorphous water has been detected in experiments. ${ }^{73-75}$ Previous studies have explored the freezing of ensembles of nanometer-sized supercooled water droplets within this temperature range ${ }^{74,76}$ or single water droplets supported on a hydrophobic substrate. ${ }^{77}$ The present study is the first known report of the homogeneous nucleation of single, levitated, submicrometer-sized water droplets within the regime known as "no-man's land."78 The "no-man's land" region in water's phase diagram is experimentally restricted due to rapid crystallization of supercooled water, ${ }^{75}$ but can be accessed by reducing particle sizes into the submicrometer size range, thus resulting in less frequent nucleation events. ${ }^{73}$

The particles for which freezing was observed in our studies ranged in size from $r=456 \mathrm{~nm}$ to $r=1956 \mathrm{~nm}$ before freezing and had freezing times ranging from $282 \mathrm{~s}$ to $976 \mathrm{~s}$ at $228 \mathrm{~K}$. Rough estimates of freezing times for supercooled water droplets at this temperature were determined using homogeneous nucleation rates for $\mathrm{H}_{2} \mathrm{O}$ aerosols between 236.37 and $237.91 \mathrm{~K}$ from EDB studies extrapolated to $228.0 \mathrm{~K} .{ }^{10}$ Applying the extrapolated nucleation rate from this previous work predicts a freezing time for a particle of $r=1$ $\mu \mathrm{m}$ in size of less than $1 \mathrm{~s}$, which is faster than our measured freezing times at $228 \mathrm{~K}$. This discrepancy highlights that future detailed studies on a large number of water droplets are critically important to assess homogeneous nucleation rates at these lower temperatures for smaller, submicrometer-sized particles.

\section{CONCLUSION AND OUTLOOK}

We have presented the design of a temperature-controlled instrument for trapping single submicrometer-sized particles. With the newly developed apparatus, we have demonstrated freezing of single, supercooled hexadecane, dodecane, and water aerosols both homogeneously and heterogeneously (via contact freezing). Evaporation of single supercooled aerosol particles has also been monitored at various temperatures. Initial measurements of the freezing times of supercooled water droplets within "no-man's land" at $228 \mathrm{~K}$ are longer than predicted freezing times extrapolated from available homogeneous nucleation rates. Systematic studies of the freezing kinetics of these supercooled particles down into the submicrometer size range will be the subject of future investigations.

With the current CPBB trap, we observed that supercooled liquid particles are sometimes lost after freezing (see Sec. III A). This has to do with the limited trapping stability of the CPBB trap. A recent theoretical study from our group shows that the combination of two perpendicularly arranged $\mathrm{CPBB}$ traps creates an optical trap that is much more stable and robust (called the quadruple Bessel beam trap). ${ }^{52}$ Implementation of this quadruple Bessel beam (QBB) trap will improve the trapping stability for smaller, submicrometer-sized supercooled liquid and solid particles, thereby increasing overall trapping time and particle retention after freezing. Another difficulty is the lack of a suitable method to accurately determine the size of small particles (see Sec. III). Standard elastic light scattering techniques can only achieve an accuracy of $\pm 100 \mathrm{~nm}$, and are limited in their utility for sizing submicrometer-sized particles due to the absence of characteristic structure in the scattering pattern at these sizes. Towards this end, we plan to implement a white light scattering system to improve size analysis of submicrometer-sized particles. We have just demonstrated at room temperature that white light scattering allows us to size particles smaller than $r=400 \mathrm{~nm}$ and in general to size particles with greater accuracy (publication in preparation). Finally, the experimental temperature range of our current apparatus is limited and spans a range from approximately room temperature $(300 \mathrm{~K})$ to $223 \mathrm{~K}$. We would like to extend this from $373 \mathrm{~K}$ to as low as $78 \mathrm{~K}$ to study single pure and mixedcomponent particles that may be relevant to planetary and lunar atmospheres to complement our previous studies of particle ensembles. ${ }^{48-51,68-70}$ Combining the more stable QBB trap and more accurate size determination with white light scattering in an apparatus that has a greater temperature range will provide the tools necessary for these future studies.

\section{ACKNOWLEDGMENTS}

We would like to thank Kerry Knox for insightful discussions. We are grateful to M. Kerallaj from the ETH LPC Machine Shop and M. Steger from the ETH LPC Electronics Shop for their expertise and assistance in constructing the apparatus. J.W.L. acknowledges funding from a Marie Curie Fellowship from the European Commission (BBOT \#331734). M.I. received funding from an NSERC postgraduate scholarship. Financial support is acknowledged from ETH and the Swiss National Science Foundation (SNSF) through Grant No. 200021_146368/1.

${ }^{1}$ B. J. Dennis-Smither, K. L. Hanford, N.-O. A. Kwamena, R. E. H. Miles, and J. P. Reid, J. Phys. Chem. A 116, 6159 (2012).

${ }^{2}$ G. Hargreaves, N.-O. A. Kwamena, Y. H. Zhang, J. R. Butler, S. Rushworth, S. L. Clegg, and J. P. Reid, J. Phys. Chem. A 114, 1806 (2010).

${ }^{3}$ T. Koop, J. Bookhold, M. Shiraiwa, and U. Pöschl, Phys. Chem. Chem. Phys. 13, 19238 (2011).

${ }^{4}$ B. Zobrist, V. Soonsin, B. P. Luo, U. K. Krieger, C. Marcolli, T. Peter, and T. Koop, Phys. Chem. Chem. Phys. 13, 3514 (2011).

${ }^{5}$ R. Power, D. L. Bones, and J. P. Reid, Proc. SPIE 8458, 845829 (2012)

${ }^{6}$ D. L. Bones, J. P. Reid, D. M. Lienhard, and U. K. Krieger, Proc. Natl. Acad. Sci. U.S.A. 109, 11613 (2012).

${ }^{7}$ H.-J. Tong, J. P. Reid, D. L. Bones, B. P. Luo, and U. K. Krieger, Atmos. Chem. Phys. 11, 4739 (2011).

${ }^{8}$ B. Krämer, M. Schwell, O. Hübner, H. Vortisch, T. Leisner, E. Rühl, H. Baumgärtel, and L. Wöste, Ber. Bunsen-Ges. Phys. Chem. 100, 1911 (1996).

${ }^{9}$ N. Hoffmann, D. Duft, A. Kiselev, and T. Leisner, Faraday Discuss. 165, 383 (2013).

${ }^{10} \mathrm{P}$. Stöckel, I. M. Weidinger, H. Baumgärtel, and T. Leisner, J. Phys. Chem. A 109, 2540 (2005).

${ }^{11}$ D. Duft and T. Leisner, Atmos. Chem. Phys. 4, 1997 (2004). 
${ }^{12}$ I. Weidinger, J. Klein, P. Stöckel, H. Baumgärtel, and T. Leisner, J. Phys Chem. B 107, 3636 (2003).

${ }^{13}$ P. Stöckel, H. Vortisch, T. Leisner, and H. Baumgärtel, J. Mol. Liq. 96-97, 153 (2002).

${ }^{14}$ H. Vortisch, B. Krämer, I. Weidinger, L. Wöste, T. Leisner, M. Schwell, H. Baumgärtel, and E. Rühl, Phys. Chem. Chem. Phys. 2, 1407 (2000).

${ }^{15}$ B. Krämer, O. Hübner, H. Vortisch, L. Wöste, and T. Leisner, J. Chem. Phys. 111, 6521 (1999).

${ }^{16}$ U. Lohmann and J. Feichter, Atmos. Chem. Phys. 5, 715 (2005).

${ }^{17}$ J. Wang, D. J. Jacob, and S. T. Martin, J. Geophys. Res.: Atmos. 113, D11207, doi:10.1029/2007JD009368 (2008).

${ }^{18}$ S. T. Martin, H.-M. Hung, R. J. Park, D. J. Jacob, R. J. D. Spurr, K. V. Chance, and M. Chin, Atmos. Chem. Phys. 4, 183 (2004).

${ }^{19}$ E. J. Davis and A. K. Ray, J. Colloid Interface Sci. 75, 566 (1980).

${ }^{20}$ E. A. Svensson, C. Delval, P. von Hessberg, M. S. Johnson, and J. B. C. Pettersson, Atmos. Chem. Phys. 9, 4295 (2009).

${ }^{21}$ A. Pluchino, J. Opt. Soc. Am. A 4, 614 (1987).

${ }^{22}$ U. K. Krieger, C. A. Colberg, U. Weers, T. Koop, and T. Peter, Geophys. Res. Lett. 27, 2097, doi:10.1029/2000GL011613 (2000).

${ }^{23}$ E. J. Davis, Langmuir 1, 379 (1985).

${ }^{24}$ K. Taji, M. Tachikawa, and K. Nagashima, Appl. Phys. Lett. 88, 141111 (2006).

${ }^{25}$ K. Anders, N. Roth, and A. Frohn, J. Geophys. Res. 101, 19223, doi:10.1029/95JD03227 (1996).

${ }^{26}$ N. Roth and A. Frohn, Atmos. Environ. 32, 3139 (1998).

${ }^{27}$ A. Ashkin and J. M. Dziedzic, Appl. Phys. Lett. 19, 283 (1971).

${ }^{28}$ C. Mund and R. Zellner, ChemPhysChem. 4, 630 (2003).

${ }^{29}$ C. Mund and R. Zellner, ChemPhysChem. 4, 638 (2003).

${ }^{30}$ H. Suzuki, Y. Matsuzaki, A. Muraoka, and M. Tachikawa, J. Chem. Phys. 136, 234508 (2012).

${ }^{31}$ S. Ishizaka, T. Wada, and N. Kitamura, Chem. Phys. Lett. 506, 117 (2011).

${ }^{32}$ K. Diehl, M. Ettner-Mahl, A. Hannemann, and S. K. Mitra, Atmos. Res. 94, 356 (2009).

${ }^{33}$ M. Ettner, S. K. Mitra, and S. Borrmann, Atmos. Chem. Phys. 4, 1925 (2004).

${ }^{34}$ K. L. Carleton, D. N. Sonnenfroh, and W. T. Rawlins, J. Geophys. Res. 102, 6025, doi:10.1029/97JD00009 (1997).

${ }^{35}$ T. Cizmar, V. Garcez-Chavez, K. Dholakia, and P. Zemanek, Proc. SPIE 5514, 643 (2004).

${ }^{36}$ V. Karasek, T. Cizmar, O. Brzobohaty, and P. Zemanek, Phys. Rev. Lett. 101, 143601 (2008).

${ }^{37}$ T. Cizmar, V. Garcez-Chavez, K. Dholakia, and P. Zemanek, Proc. SPIE 5930, 59300X (2005).

${ }^{38}$ T. Cizmar, V. Kollarova, Z. Bouchal, and P. Zemanek, New J. Phys. 8, 43 (2006).

${ }^{39}$ A. E. Carruthers, J. S. Walker, A. Casey, A. J. Orr-Ewing, and J. P. Reid, Phys. Chem. Chem. Phys. 14, 6741 (2012).

${ }^{40}$ M. D. Summers, J. P. Reid, and D. McGloin, Opt. Express 14, 6373 (2006).

${ }^{41}$ H. Meresman, J. B. Wills, M. D. Summers, D. McGloin, and J. P. Reid, Phys. Chem. Chem. Phys. 11, 11333 (2009).

${ }^{42}$ M. I. Cotterell, B. J. Mason, A. E. Carruthers, J. S. Walker, A. J. Orr-Ewing, and J. P. Reid, Phys. Chem. Chem. Phys. 16, 2118 (2014).
${ }^{43}$ J. W. Lu, A. M. J. Rickards, J. S. Walker, K. J. Knox, R. E. H. Miles, J. P. Reid, and R. Signorell, Phys. Chem. Chem. Phys. 16, 9819 (2014).

${ }^{44}$ M. N. Eremenko, A. Y. Zasetsky, C. D. Boone, and J. J. Sloan, Geophys. Res. Lett. 32, L15S07, doi:10.1029/2005GL022428 (2005).

${ }^{45}$ D. Rosenfeld and W. L. Woodley, Nature (London) 405, 440 (2000).

${ }^{46}$ L.-Y. He, X.-F. Huang, L. Xue, M. Hu, Y. Lin, J. Zheng, R. Zhang, and Y.-H. Zhang, J. Geophys. Res. 116, D12304, doi:10.1029/2010JD014566 (2011).

${ }^{47}$ B. P. Lee, J. L. Yong, J. Z. Yu, P. K. K. Louie, and C. K. Chan, J. Geophys. Res. 118, 8625, doi:10.1002/jgrd.50658 (2013).

${ }^{48}$ M. Isenor, R. Escribano, T. C. Preston, and R. Signorell, Icarus 223, 591 (2013).

${ }^{49}$ E. K. Lang, K. J. Knox, and R. Signorell, Planet. Space Sci. 75, 56 (2013).

${ }^{50}$ E. K. Lang, K. J. Knox, C. C. Wang, and R. Signorell, Planet. Space Sci. 59, $722(2011)$.

${ }^{51}$ C. C. Wang, E. K. Lang, and R. Signorell, Astrophys. J. Lett. 712, L40 (2010).

${ }^{52}$ I. Thanopulos, D. Luckhaus, T. C. Preston, and R. Signorell, J. Appl. Phys. 115, 154304 (2014)

${ }^{53}$ C. F. Bohren and D. R. Huffman, Absorption and Scattering of Light by Small Particles (Wiley-VCH Mörlenbach, 2004).

${ }^{54}$ C. Wolfharth, in Springer Materials - The Landolt-Börnstein Database, edited by M. D. Lechner (Spring-Verlag Berlin, 2008).

${ }^{55}$ CRC Handbook of Chemistry and Physics, 94th ed., edited by D. R. Lide (Taylor and Francis Group, Boca Raton, FL, 2013).

${ }^{56}$ R. C. Millard, and G. Seaver, Deep-Sea Res. 37, 1909 (1990).

${ }^{57}$ D. Duft and T. Leisner, Int. J. Mass Spectrom. 233, 61 (2004).

${ }^{58}$ L. Carroll and M. Henry, Appl. Opt. 41, 1330 (2002).

${ }^{59}$ G. S. Parks and G. E. Moore, J. Chem. Phys. 17, 1151 (1949).

${ }^{60}$ H. R. Pruppacher, J. Atmos. Sci. 52, 1924 (1995).

${ }^{61}$ O. F. Sigurbjörnsson and R. Signorell, Phys. Rev. E 77, 051601 (2008).

${ }^{62}$ C. Mund and R. Zellner, J. Mol. Struct. 661-662, 491 (2003).

${ }^{63}$ E. B. Sirota and A. B. Herhold, Science 283, 529 (1999).

${ }^{64}$ X.-Y. Liu and P. Bennema, J. Cryst. Growth 135, 209 (1994).

${ }^{65}$ Y. S. Djikaev and E. Ruckenstein, J. Phys. Chem. A 112, 11677 (2008).

${ }^{66}$ S. C. Nyberg and F. M. Pickard, Acta Crystallogr., Sect. B: Struct. Crystallogr. Cryst. Chem. B32, 2289 (1976).

${ }^{67}$ V. P. Modak, H. Pathak, M. Thayer, S. J. Singer, and B. E. Wyslouzil, Phys. Chem. Chem. Phys. 15, 6783 (2013).

${ }^{68}$ O. F. Sigurbjörnsson and R. Signorell, Phys. Chem. Chem. Phys. 10, 6211 (2008).

${ }^{69}$ E. K. Lang, K. J. Knox, T. Momose, and R. Signorell, J. Phys. Chem. A 117, 11745 (2013)

${ }^{70}$ C. C. Wang, S. K. Atreya, and R. Signorell, Icarus 206, 787 (2010).

${ }^{71}$ N. J. Bacon and B. D. Swanson, J. Atmos. Sci. 57, 2094 (2000)

${ }^{72}$ H. Kanno, R. J. Speedy, and C. A. Angell, Science 189, 880 (1975).

${ }^{73}$ T. Li, D. Donadio, and G. Galli, Nat. Commun. 4, 1887 (2013).

${ }^{74}$ A. Manka, H. Pathak, S. Tanimura, J. Wölk, R. Strey, and B. E. Wyslouzil, Phys. Chem. Chem. Phys. 14, 4505 (2012).

${ }^{75}$ E. B. Moore and V. Molinero, J. Chem. Phys. 132, 244504 (2010).

${ }^{76}$ J. Huang and L. S. Bartell, J. Phys. Chem. 99, 3924 (1995).

${ }^{77}$ B. J. Murray, S. L. Broadley, T. W. Wilson, S. J. Bull, R. H. Wills, H. K. Christenson, and E. J. Murray, Phys. Chem. Chem. Phys. 12, 10380 (2010).

${ }^{78}$ O. Mishima and H. E. Stanley, Nature (London) 396, 329 (1998). 